\title{
Application of Molecular Biological Biomarkers to Endocrine Disruption Studies
}

\author{
Dongmei Guo ${ }^{1}$, Jing $\mathrm{Qiu}^{2}$, Yun $\mathrm{Li}^{2}$, Guiling Yang${ }^{1}$ and Yongzhong Qian ${ }^{2 *}$ \\ ${ }^{1}$ Institute of Quality and Standard for Agro-Products, China \\ ${ }^{2}$ Key Laboratory of Agro-Product Quality and Safety of Ministry of Agriculture, China
}

*Corresponding author: Yongzhong Qian, Key Laboratory of Agro-Product Quality and Safety of Ministry of Agriculture, Institute of Quality Standards and Testing Technology for Agro-Products, Chinese Academy of Agricultural Sciences, China

\begin{abstract}
ARTICLE INFO
Received: 慧 October 28, 2019

Published: November 18, 2019

Citation: Dongmei Guo, Jing Qiu, Yun Li, Guiling Yang, Yongzhong Qian. Application of Molecular Biological Biomarkers to Endocrine Disruption Studies. Biomed J Sci \& Tech Res 22(5)-2019. BJSTR. MS.ID.003829.
\end{abstract}

\section{ABSTRACT}

In the natural environment, Endocrine Disrupting Chemicals (EDCs)may bring about great impact on the ecological environment and human health, and people have begun to understand the importance of studying the potential risks resulted from the pollution of EDCs. Molecular biomarkers provide an innovative technique for the screening and research of EDCs. This review included the methods of studying estrogenic biomarkers, thyroid hormone biomarkers, reproduction hormone biomarkers and latest progress of using EDCs biomarkers, aiming at ffective screening and detection of contaminants in EDCs.

Abbreviations: EDCs: Endocrine Disrupting Chemicals; HPT: Hypothalamic-PituitaryThyroid; HPG: Hypothalamus-Pituitary-Gonad; VTG: Vitellogenin; ER: Estrogenic Receptors; FSH: Follicle Stimulating Hormone; LH: Luteinizing Hormone; TCDD: Tetrachlorodibenzo-Para-Dioxin; ZFL: Zebrafish Liver; TRs: Thyroid Receptors; SPs: Synthetic Pyrethroids; TH: Thyroid Hormone; PER: Perchlorate; GnRH: Gonadotropin Releasing Hormone; PFAAs: Perfluoroalkyl Acids; CAT: Catalase; SOD: Superoxide Dismutase; GPx: Glutathione Peroxidase; GSH: Glutathione; MDA: Malondialdehyde; T: Testosterone

\section{Introduction}

Endocrine Disrupting Chemicals (EDCs), also called environmental hormones, were first proposed in the book "Our Stolen Future" by a journalist Dianne Dumanoski [1]. EDCs are a group of xenobiotics greatly impacting the environment. As substances similar to endocrine hormones in living organisms, EDCs disrupt the normal secretion of hormones in bodies when entering animal and human bodies. This way, physiological disorders will occur, impacting the reproductive, nervous and endocrine systems of animals and humans or even causing carcinogenicity. Specifically, aquatic organisms are directly exposed to pollutants, and the endocrine interference impact on them is even greater [2-4]. EDCs feature various types, continuous production, diversified forms and bioaccumulation. In recent years, EDCs have become the most severe pollution issue after ozone depletion and global warming and attracted attention of many countries and international organizations [5].

Fish is often considered one of the model organisms for endocrine disruptor evaluation because endocrine disruptors may interfere with reproduction hormones in fish. First, sexual differentiation is unstable in fish, implying that during the sexual differentiation phase of this vertebrate, it may be disrupted by external hormones, or its sex may be even changed [6]. Second, aquatic ecosystem is under attack from various pollutants, and fish is directly exposed to industrial, agricultural and municipal wastewater and pollutants. Current evidence showed that longterm exposure to EDCs would affect reproduction and population of fishes [7]. Endocrine disruptors may affect important hormones or receptors by disrupting the endocrine pathway along the 
Hypothalamus-Pituitary-Gonad (HPG) axis of fish, which finally has an impact on animal reproduction. EDCs may also disrupt the Hypothalamic-Pituitary-Thyroid (HPT) axis of fish, interfere with thyroid hormone synthesis, transport and combination, destabilize the thyroid hormone environment, and are therefore harmful to the growth and development of fish. Environmental pollutants can affect the synthesis of steroid hormones and serve as an endocrine disruptor through a receptor independent pathway. Molecular biological technologies, such as -omics and transgenic techniques, provide a reliable approach to evaluate the threats from endocrine disruptors in environmental pollutants. They may also reveal the pollutant mechanism. In addition, specific genes in fish also help determine biomarkers as endocrine disruptors [8].

Biomarkers can be defined as an indicator for the level of exposure to and/or toxic effects of one or more chemical pollutants, which affect organisms at biological levels such as molecular, cell, and individual levels and cause measurable changes in tissues, biological fluids, or biochemical, cell, physiological, or behavioral features of organisms [9]. Some biomarkers can predict the effects of reproductive or other endocrine disruption based on the linkage between endocrine disruption and endpoints of molecular biological experiments. Therefore, biomarkers for EDCs now become a study focus after the establishment of highly efficient experimental methods reflecting the actual situation in organisms with a low cost and little or no damage. Biomarkers are widely applied to and play a significant role in endocrine disruption researches. Due to the diversified functions of EDCs, the types of biomarkers become increasingly abundant, with continuous emergence of a large number of new biomarkers.

\section{Estrogenic Biomarkers}

A distinctive feature of EDCs is their estrogenic effect. By emulating endogenous estrogens, EDCs lead to feminization of male individuals. Many synthetic compounds with different structures have estrogen or antiestrogen effects [10]. According to multiple studies, Vitellogenin (VTG) in adult fish may function as an ideal estrogenic biomarker. VTG is a precursor protein of egg yolk and is usually synthesized as a result of endogenous estrogen stimulation. VTG generally exists in the plasma of female fish. Though male fish also has VTG genes, VTG proteins cannot be autonomously synthesized in male fish in the absence of estrogen. However, stimulated by exogenous estrogen, VTG proteins may be synthesized in male fish. VTG is specific and sensitive to estrogen stimuli and has a high expression level in a number of organisms. Therefore, it can be used as an excellent estrogenic biomarker [11]. Recently, VTG expression was employed to evaluate the estrogenic effect of environmental chemicals. As compared to protein-based detection, VTG-based detection has a comparable sensitivity. Therefore, VTG can be employed as a biomarker to detect estrogenic effect at an early phase [12,13]. At present, VTG genes in nearly 20 kinds of fish have been successfully cloned. Therefore, VTG may promote the applications of this biomarker in a wider range.
Estrogen plays a critical role in regulating the reproductive, developmental, and neuroendocrine systems of fish. In the study of the estrogenic effect, some other biomarkers have been discovered. Estrogenic Receptors (ERs) and aromatase are essential for synthesis, secretion and physiological functions of estrogen. Estrogenic effect is produced when compounds simulate estrogen to combine with ERs and activate the ER genes. In bony fish, there are mainly two estrogenic receptor subtypes $\mathrm{ER} \alpha$ and $\mathrm{ER} \beta$, which activate gene expression by binding to specific response elements $[14,15]$. In addition, estrogenic effect may be indicated by an increase in the level of free endogenous estrogens in plasma caused by the competitive binding of exogenous chemical hormones to globulin [16]. The antiestrogen effect implies that compounds have no or little estrogen activity but could perform competitive binding with ERs. In addition, the antiestrogenic effect influence, the expression and activity of the cytochrome p450a19 enzyme (aromatase).

Villeneuve et al. studied the impact of fadrozole on the expression of genes related to reproduction in the HPG axis and liver of fathead minnow. In the perspective of systems biology, they established an evaluation system for the impact of pollutants on the HPG and determined the association between gene expression and experimental endpoints of reproductive biology. This method aimed at the functions of multiple important organs instead of only one organ such as the gonad, which helped understand the influence and interaction between pollutants and the entire endocrine system of fish [17]. Moreover, this approach depicted the molecular function model and helped establish a relationship reflecting the influence of molecular biological changes on organism reproduction. Zhang et al. explored the impacts of prochloraz and ketoconazole on the expression of reproduction-related genes in the HPG axis and liver of medaka. They found a linear relationship between the expressions of six genes in the liver and spawning amounts. Therefore, gene expression amounts could be used to speculate on the biological experiment endpoint of fish in the view of ecology [18].

Dongmei et al. [19] studied the estrogen-related genes in the HPG axis of the zebrafish embryo, or more specifically estrogenassociated biomarkers including VTG1, VTG2, ER $\alpha, \mathrm{ER} \beta 1, \mathrm{ER} \beta 2$, CYP19a1a and CYP19a1b, based on which the joint effect of compound estrogen involving cypermethrin, malathion and prochloraz on zebrafish could be determined. The test results demonstrated that within a period under a certain drug amount, the estrogenic effect was effectively enhanced with binary pesticides of cypermethrin and malathion or malathion and prochloraz when compared with the treatment of a single chemical. In addition, the expression amounts of ER $\alpha, E R \beta 1, E R \beta 2, C Y P 19 b$ and CYP19a were significantly changed.

Jinhua et al. [20] used molecular biomarkers such as estrogen and thyroid hormone to study the endocrine disruption of acetochlor at the early life stage of zebrafish. After zebrafish was exposed to acetochlor at the concentrations of 50,100 and $200 \mu \mathrm{g} / \mathrm{L}$, the HPG/ 
HPT-associated genes in zebrafish, such as VTG1, ER $\beta 1$, CYP19a and $\mathrm{TR} \alpha$, and many key genes in the apoptosis pathway, including $\mathrm{Bcl} 2$, Bax, P53 and Cas8, underwent significant changes. The results deomonstrated that acetochlor could induce oxidative stress and apoptosis at the developmental stage of zebrafish and affect the immune and endocrine systems of zebrafish. Yingying C. et al. [21] reported the estrogenic effects of 2,3,7,8-TetrachlorodibenzoPara-Dioxin (TCDD) on the gene expressions of the Zebrafish Liver (ZFL) cell lines, and zebrafish embryo, larva, juvenile and adult zebrafish livers, both independently and when combined with Cd 2+. In ZFL cells, mRNA expression of VTG1 was significantly suppressed by $\mathrm{Cd} 2+$ but was not affected by TCDD. In ZFL cells, the expressions of ER $\alpha, E R \beta 1, E R \beta 2$ and GPER were insignificantly changed. They also conducted a study on the VTG1 promoter deletion mutant and found no reaction in the presence of TCDD or $\mathrm{Cd} 2+$. However, following co-transfection with a VTG1 promoterluciferase construct to the ER $\alpha, E R \beta 1, E R \beta 2$ and GPER expression vectors, decreased luciferase activity was observed in the ER $\alpha$ cotransfection group after treatment with $\mathrm{Cd} 2+$, suggesting that ER $\alpha$ participated in VTG1 transcriptional regulation and was affected by $\mathrm{Cd} 2+$. The regulation of these genes at the mRNA level varied in male and female zebrafish livers at different developmental phases.

\section{Thyroid Hormone Biomarkers}

Another prominent feature of EDCs lies in the thyroid effect. Thyroid hormone helps maintain the normal physiological conditions of vertebrates and is considered the most important factor in controlling their growth, development and behaviors. In bony fish, thyroid hormone also plays a vital role in regulating metabolism. The synthesis and release of thyroid hormones are controlled by the HPT axis. Chemicals that disrupt one or more points in the HPT axis may affect the thyroid hormone function, and finally impact the growth and development of animals. Thyroid-disrupting chemicals may interfere with different regulation pathways, especially synthesis and metabolism (binding) of thyroid hormones, for example, synthesis through iodinated thyroglobulin or changing T4 into T3 with higher physiological activity. They may also disrupt plasma protein binding or combine with Thyroid Receptors (TRs) [22]. After combining with ligands, inhibitory factors activated proteins and TR homo-/hetero-dimers or retinoic X to induce the expression of target genes of thyroid response elements, such as phosphoenolpyruvate carboxykinase or 5'-diodinase [23]. In bony fish, there were two TR isomers ( $\alpha$ and $\beta$ ) and splice variants [23]. It was difficult to identify insignificant impacts of thyroid hormone on organisms in laboratories. However, molecular markers could serve as an indicator and prediction signal to overcome the difficulty in short-period experiments. Molecular biomarkers, which are similar to estrogen regulation genes such as VTG, could be used as potential sensitive biomarkers for thyroid hormones.

Wenqing et al. [24] studied the disruption of Synthetic Pyrethroids (SPs) on the HPT axis of zebrafish embryo under acute exposure conditions. SPs might damage the thyroid endocrine system of mammals. 1, 3 and $10 \mu \mathrm{g} / \mathrm{L}$ of bifenthrin or cyhalothrin might influence the levels of T4 or T3. In addition, the genes such as CRH, TSH $\beta$, TTR, UGT1ab, Pax8, Dio 2 and TR $\alpha$ in the HPT axis of zebrafish were significantly upregulated as induced by bifenthrin. Cyhalothrin could influence different test genes and significantly induce the expressions of TTR, Pax8, Dio2 and TR $\alpha$. However, the Dio1 gene was greatly suppressed. At the atomic level, compared with cyhalothrin, the combined protein of bifenthrin and TR $\alpha$ had more powerful influence on the HPT signal conduction. According to Shi et al. [25], PFOS had apparent development toxicity effect on zebrafish embryo. The expressions of marker genes hhex and pax8 for early thyroid development were significantly upregulated, indicating possible thyroid development toxicity. The study on the thyroid toxicity of PCB126 to salmon [26] revealed that the levels of T4 in the salmon plasma and T4 glucosylation significantly rose, resulting in swelling of the thyroid epithelial cells. As the herbicides used with the largest amounts in China, acetochlor decreased the Thyroid Hormone (TH) level according to the Crum's study on xenopus laevis, which impacted the thyroid functions. The author explained the mechanism at the molecular level [27].

Xuesong et al. [28] studied the thyroid endocrine toxicity of 2,2',4',4-tetrabromodiphenyl ether (BDE-47) and Perchlorate (PER) based on the TH-mediated pathway after zebrafish embryo was exposed to them for 14 days. The test results indicated that when compared with exposure to only BDE-47, combined exposure to BDE-47 (10 $\mu \mathrm{g} / \mathrm{L})$ and PER (3.5 mg/L) could significantly upregulate the genes NIS and Nkx2.1a that participated in TH synthesis, which highly downregulated HPT axis ( $\mathrm{CRH}$ and TSH) mediated by expression of regulation-related genes. Compared to single BDE-47 exposure, combined exposure to BDE- 47 and PER could greatly lift the TG gene and protein levels, while significantly downregulated the TTR gene and protein levels. In addition, exposure to the compounds of BDE-47 and PER greatly decreased the T4 level, indicating that the damage to the BDE-47 thyroid was increased by PER. The results helped explain the complicated chemical interactions and the molecular mechanisms of the two disruptors.

\section{Reproduction Hormone Biomarkers}

The HPG axis controls the reproduction of fish species among all vertebrates. During the fish spawning season, external factors such as light and temperature of water affect the gonad recovery and brain maturation. Signals from the brain control the Gonadotropin Releasing Hormone (GnRH), and GnRH stimulates the hypothalamus-pituitary, which secretes gonadotropin, including Luteinizing Hormone (LH) and Follicle Stimulating Hormone (FSH). Then, LH and FSH, in return, control the production and secretion of steroid hormones from the gonad. Hormones, including the main hormones and sex inducing hormones of $17 \beta$-estradiol and testosterone in female individuals, as well as testosterone and 11-keto-testosterone in male individuals, initiate changes in secondary sexual characteristics, in addition to the development and maturation of sperm and eggs. In different physiological 
stages, reproduction hormones regulated the secretion of pituitary gonadotropin hormones according to the positive or negative feedback [29]. The HPG axis provided several potential modes, which affected the synthesis and secretion of EDCs. By binding to hormone receptors or changing the usability of endogenous hormones, EDCs exerted their disruption function through the mediation of steroid hormones or similar elements. Therefore, biomarkers directly reflected the level of steroid hormones and could function as a widely applied molecular indicator of endocrine disruptors. VTG synthesis and secretion were induced in livers by cyclic estrogen, and $17 \beta$-estradiol was dedicated to the identification of exogenous estrogen [30].

Wei Zh et al. [31] studied the chronic reproductive toxicity and possible mechanism of Perfluoroalkyl Acids (PFAAs) after adult zebrafish (Danio rerio) was exposed to different concentrations (0.01, 0.1 and $1 \mathrm{mg} / \mathrm{L}$ ) of PFAAs for 180 days. The disrupted expression of genes, such as ER $\alpha$, ER $\beta$, FSHR, LHR, StAR and $17 \beta H S D$, indicated possible interference of PFNA on the HPGL axis function and sex hormone synthesis. PFNA interfered with the HPGL axis function and sex hormone synthesis by disturbing the expression of genes in the HPGL axis, which led to estrogenic effects such as significantly increased VTG content in males and increased E2 levels in both genders. The results of this study provided a basis for research on the potential risks of this ubiquitous and persistent contaminant in aquatic ecosystems worldwide.

Kyunghee J et al. [32] carried out a study on the impact of non-steroidal anti-inflammatory drugs (NSAIDs) on the PHG genes of zebrafish and their reproduction toxicity. This study found that ibuprofen and mefenamic acids significantly increased the concentrations of $17 \beta$-estradiol and testosterone in females, while decreased those of testosterone among male fish after the adult zebrafish was exposed to the drugs for 14 days. Significant upregulation of FSH $\beta$, LH $\beta$, FSHR and LHR was observed in females, whereas downregulation was observed in males exposed to NSAIDs. After adult zebrafish pairs were exposed to ibuprofen for 21 days, and the egg production was significantly decreased at $1 \mu \mathrm{g} / \mathrm{L}$ ibuprofen, parental exposure resulted in delayed hatching even if they were transferred to clean water for hatching. The results demonstrated that ibuprofen could modulate hormone production and related gene transcription of the HPG axis in a sex-dependent way, which could cause adverse effects on the reproduction and development of offspring.

Qun-Fang Z et al. [33] conducted research on the reproductive toxicity of zebrafish under exposure to inorganic mercury (Hg). After adult zebrafish was exposed to 0 (control), 15 and $30 \mu \mathrm{g}$ of $\mathrm{Hg}$ $\mathrm{L}$ (added as mercuric chloride, $\mathrm{HgCl} 2$ ) for 30 days, the activities and mRNA levels of antioxidant enzymes (catalase (CAT), Superoxide Dismutase (SOD) and Glutathione Peroxidase (GPx)) as well as the contents of Glutathione (GSH) and Malondialdehyde (MDA) were changed. In females, although the ovarian $17 \beta$-estradiol (E2) content remained relatively stable, significant downregulation of LHR, GNRH2, GNRH3, LHR and EAR were observed. In males, Testosterone ( $\mathrm{T}$ ) levels in the testis significantly decreased after $\mathrm{Hg}$ exposure, accompanied by down-regulated expression of GNRH2, GNRH3, FSH $\beta$ and LH $\beta$ in the brain as well as FSH $\beta, \mathrm{LH} \beta, \mathrm{AR} \beta$, CYP17 and CYP11b in the testis. Thus, our study indicated that waterborne inorganic $\mathrm{Hg}$ exposure altered sex hormone levels by disrupting the transcription of related HPG-axis genes, which could subsequently impair fish reproduction.

\section{Conclusion}

EDCs may bring about great impact on the ecological environment and human health, and people have begun to understand the importance of studying the potential risks resulted from the pollution of EDCs. It is important to perform researches on the toxicity of EDCs, especially the joint toxic effects caused by long-term exposure to endocrine disruptors at low dosage. The biological screening methods of EDCs, either in vivo or in vitro, require a high cost. Moreover, damages will be present in in-vivo biological tests, and significantly different results will occur in invitro tests when compared to in-vivo tests. Molecular biomarkers provide an innovative technique for the screening and research of EDCs. One of the advantages of this method is the low cost and effective reflection of actual conditions inside organisms. Another advantage lies in its sensitivity to the molecular endpoints in aromatase inhibitors, hormone levels and expressions of various genes. After fish is exposed for two to three weeks to chemicals at the concentration that can influence the reproduction, the molecular endpoints of the tests can be sensitively influenced. Using molecular endpoints is a relatively simple screening method for the study of endocrine disruption effects. Therefore, it is extremely important to use molecular biological technologies to establish a fast, reliable, specific and sensitive screening method for effective screening and detection of contaminants in EDCs.

\section{Acknowledgement}

The research was supported by Science and Technology Innovation Program of Chinese Academy of Agricultural Sciences and Zhejiang Province Major Bidding Project (Grant No. 2014C02002).

\section{References}

1. Kavlock RJ, Daston GP, De Rosa C, Fenner Crisp P, Gray LE, et al. (1996) Research needs for the risk assessment of health and environmental effects of endocrine disruptors: A reportor of the U.S.EPA-Sponsored worksho. Environ health perspect 104: 715-740.

2. Rhind SM (2005) Are endocrine disrupting compounds a threat to farm animal health, welfare and productivity? Reprod Domest Anim 40(4): 282-290.

3. Patrick L (2009) Thyroid disruption: Mechanism and clinical implications in human health. Altern Med Rev 14(4): 326-346.

4. Eva RK, Monica SR, Imon R (2015) A review on endocrine disruptors and their possible impacts on human health. Environ Toxicol Pharmacol 40(1): 241-258.

5. Zhang J, Guo S (2012) Influence of Environmental Estrogens on Immune Systems of Fish. Sci Technol Infor (5): 266. 
6. Francis RC (1992) Sexual lability in teleosts: Developmental factors. Q Rev Biol 67(1): 1-18.

7. Kidd KA, Blanchfield PJ, Mills KH, Palace VP, Evans RE, et al. (2007) Collapse of a fish population after exposure to a synthetic estrogen. Proc Natl Acad Sci U. S. A 104(21): 8897-8901.

8. Muncke J, Eggen RIL (2006) Vitellogenin 1 mRNA as an early molecular biomarker for endocrine disruption in developing zebrafish (Danio rerio). Environ Toxicol Chem 25(10): 2734-2741.

9. Vander VK, Keil D, Moens LN (2006) Effects of theantide Pressant mianser in inzebrafish: Moleeular markers of endoerinedisrt IPtion. Chemos Phere 65: 1836-1845.

10. Jobling S, Reynolds T, White R, Parker MG, Sumpter JP (1995) A variety of environmental persistent chemicals, including some phthalate plasticizers, are weakly estrogenic. Environ Health Perspect 103(6): 582-587.

11. Wahli W, DawidI B, Ryffel GU, Weber R (1981) Vitellogenesis and the vitello genin gene family. Seienee 212(4492): 298-304.

12. Tyler CR, Sum Pter JP, Witthames PR (1990) The dynamies of ooeyte growth during vitellogenes is in the rainbowtrout (Oneorhynehus mykiss). Biol Reprod 43(2): 202-209.

13. Thomas Jones E, Thorpe K, Harrison N, Thomas G, Morris C, et al (2003) Dynamieso festrogen biomarker responses in rainbow trout exposedto17p-estradioland17a-ethinylestradiol. Environ Toxicol Chem 22(12): 3001-3008.

14. Hawkins MB, Thomas P (2004) The unusual binding properties of the third distinct teleost estrogen receptor subtype ERbetaa are accompanied by highly conserved amino acid changes in the ligand binding domain. Endocrinology 145(6): 2968-2977.

15. Menuet A, Pellegrini E, Anglade I, Blaise O, Laudet V, et al. (2002) Molecular characterization of three estrogen receptor forms in zebrafish: Binding characteristics transactivation properties, and tissue distributions. Biol Reprod 66(6): 1881-1892.

16. Villeneuve DL, Larkin P, Knoebl I (2007) Development and validation of a 2,000-gene microarray for the fathead minnow (Pimephales promelas). Environ Sci Technol 41: 321-330.

17. Zhang X, Hecker M, Tompsett AR, Park JW, Jones PD, et al. (2008) Responses of the Medaka HPG Axis PCR Array and Reproduction to Prochloraz and Ketoconazole. Environ Sci Technol 22: 8614-8614.

18. Guo D, Wang Y, Qian Y, Chen C, Jiao B, et al. (2017) Joint acute and endocrine disruptive toxicities of malathion, cypermethrin and prochloraz to embryo-larval zebrafish, Danio rerio. Chemosphere 166 : 63-71.

19. Jiang J, Wu S, Liu X, Wang Y, An X, et al. (2015) Effect of acetochlor on transcription of genes associated with Oxidative stress, apoptosis, immunotoxicity and endocrine disruption in the early life stage of zebrafish. Environ Toxicol Pharmacol 40(2): 516-523.
20. Chen YY, Chan KM (2016) Regulation of vitellogenin (vtg1) and estrogen receptor (er) gene expression in zebrafish (Danio rerio) following the administration of $\mathrm{Cd} \mathrm{2+}$ and 2,3,7,8- tetrachlorodibenzo-p-dioxin (TCDD). Chemosphere 147: 467-476.

21. Brown SB, Adams BA, Cyr DG, Eales J G (2004) Contaminant effects on the teleost fish thyroid. Environ Toxicol Chem 23(7): 1680-1701.

22. Jakobs TC, Schmutzler C, Meissner J, Kohrle J (1997) The promoter of the human type I 5'-deiodinase gene-mapping of the transcription start site and identification of a DR+4 thyroid-hormone-responsive element. Eur J Biochem 247(1): 288-297.

23. Marca Pereira ML, Eppler E, Thorpe KL, Wheeler JR, Burkhardt Holm P (2011) Molecular and cellular effects of chemicals disrupting steroidogenesis during early ovarian development of brown trout (Salmo trutta fario). Environ Toxicol 29(2): 199-206.

24. Tu W, Xu C, Lu B, Lin C, Wu Y, et al. (2016) Acute exposure to synthetic pyrethroids causes bioconcentration and disruption of the hypothalamus-pituitary-thyroid axis in zebrafish embryos. Sci Total Environ 542: 876-885.

25. Shi XJ, Du Y, Lam PK, Wu KS, Zhou B (2008) Developmental toxicity and alteration of gene expression in zebrafish embryos exposed to PFOS. Toxicol Appl pharmacol 230(1): 23-32.

26. Shi XJ, Liu CS, Ke Y, Jun D, Yu LQ et al. (2009) Toxicological Research on Environmental Endocrine Disruptors. Progress Chem 21(2/3): 341-342.

27. Zhao X, Wang S, Li D, You H, Ren X (2013) Effects of perchlorate on BDE47-induced alteration thyroid hormone and gene expression of in the hypothalamus-pituitary-thyroid axis in zebrafish larvae. Environ Toxicol Pharmacol 36(3): 1176-1185.

28. Peter RE, Yu KL (1997) Neuroendocrine regulation of ovulation in fishes: Basic and applied aspects. Rev Fish Biol Fisheries 7(2): 173-197.

29. Schulz RW, Goos HJT (1999) Puberty in male fish: Concepts and recent developments with special reference to the African catfish (Clarias gariepinus). Aquaculture 177: 5-12.

30. Tyler CR, Eerden VD, Jobling S, Panter G, Sumpter JP (1996) Measurement of vitellogenin, a biomarker for exposure to oestrogenic chemicals, in a wide variety of cyprinid fish. J Comp Physiol B 166(7): 418-426.

31. Wei Z, Nan S, Minhui W, Hongxia Z, Jiayin D (2016) Zebrafish reproductive toxicity induced by chronic perfluorononanoate exposure. Aquat Toxicol 175: 269-276.

32. Ji K, Liu X, Lee S, Kang S, Kho Y, et al. (2013) Effects of non-steroidal anti-inflammatory drugs on hormones and genes of the hypothalamicpituitary-gonad axis, and reproduction of zebrafish. J Hazard Mater 15: 254-255.

33. Zhang QF, Li YW, Liu ZH, Chen QL (2016) Reproductive toxicity of inorganic mercury exposure in adult zebrafish: Histological damage, oxidative stress, and alterations of sex hormone and gene expression in the hypothalamic-pituitary-gonadal axis. Aquat Toxicol 177: 417-424.
ISSN: 2574-1241

DOI: 10.26717/BJSTR.2019.22.003829

Yongzhong Qian. Biomed J Sci \& Tech Res

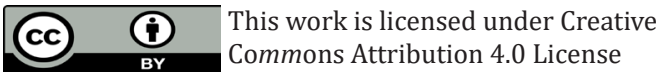

Submission Link: https://biomedres.us/submit-manuscript.php

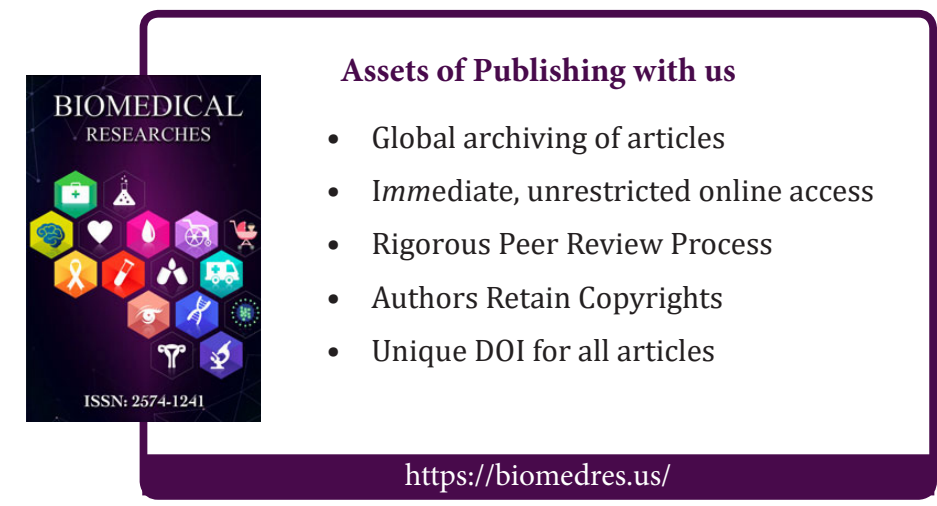

